\title{
VERTEX BARYCENTER OF GENERALIZED ASSOCIAHEDRA
}

\author{
VINCENT PILAUD AND CHRISTIAN STUMP
}

(Communicated by Jim Haglund)

ABstract. We show that the vertex barycenter of generalized associahedra and permutahedra coincide for any finite Coxeter system.

\section{INTRODUCTION}

Generalized associahedra were originally defined and studied by S. Fomin and A. Zelevinsky FZ03b and by F. Chapoton, S. Fomin, and A. Zelevinsky [CFZ02]. These polytopes realize finite type cluster complexes [FZ03a]. More general polytopal realizations of these simplicial complexes were later constructed by C. Hohlweg, C. Lange, and H. Thomas in [HLT11] by removing boundary hyperplanes from Coxeter permutahedra. This construction is based on Cambrian fans which were defined and studied by N. Reading and D. Speyer [Rea06, Rea07, RS09]. Recently S. Stella extended in Ste13 the approach of [CFZ02 and showed that the resulting realizations of generalized associahedra coincide with those of [HLT11. In [PS11, we provided a new approach to generalized associahedra using brick polytopes for spherical subword complexes. We use this latter approach to prove that the vertex barycenters of all $c$-associahedra coincide with the vertex barycenter of the underlying permutahedron.

Theorem 1.1. Let $(W, S)$ be a finite Coxeter system, let $c \in W$ be a Coxeter element, and let $\operatorname{Asso}_{c}^{u}(W)$ be the c-associahedron obtained from a fairly balanced $W$-permutahedron $\operatorname{Perm}^{u}(W)$ by removing all boundary hyperplanes not containing a c-singleton. Then the vertex barycenters of $\operatorname{Asso}_{c}^{u}(W)$ and $\operatorname{Perm}^{u}(W)$ coincide.

This property was observed by F. Chapoton for Loday's realization of the classical associahedron [Lod04]. The balanced case in types $A$ and $B$ was conjectured by C. Hohlweg and C. Lange in [HL07 and proven by C. Hohlweg, J. Lortie, and A. Raymond in [HLR10] using an orbit refinement of Theorem 1.1 in these types. The general balanced case was conjectured by C. Hohlweg, C. Lange, and H. Thomas in HLT11. This conjecture and the mentioned orbit refinement were again discussed in Hoh12. We will as well settle this orbit refinement in Section 3.4.

Finally, we want to remark that there is a naive approach to Theorem [1.1. Namely, one might hope that one can partition the vertices of the permutahedron $\operatorname{Perm}^{u}(W)$ in such a way that the sum of the vertices in each part corresponds to a vertex of the $c$-associahedron $\operatorname{Asso}_{c}^{u}(W)$. There would even be a natural candidate

Received by the editors October 16, 2012 and, in revised form, September 9, 2013.

2010 Mathematics Subject Classification. Primary 52B15; Secondary 13F60, 52B05.

The first author was supported by the Spanish MICINN grant MTM2011-22792, by the French ANR grant EGOS 12 JS02 002 01, and by the European Research Project ExploreMaps (ERC StG 208471). 
for this partition coming from the theory of Cambrian lattices [Rea07]. This approach trivially works for dihedral types, but it already turns out to fail in type $A_{3}$.

\section{Generalized associahedra}

2.1. Finite Coxeter groups. Consider a finite Coxeter system $(W, S)$, acting on a real Euclidean vector space $V$ of dimension $|S|=n$. The Coxeter arrangement is the collection of all reflecting hyperplanes in $V$. It decomposes $V$ into open polyhedral cones whose closures are called chambers. The Coxeter fan is the polyhedral fan formed by these chambers and all their faces. We denote by $\mathcal{C}$ the fundamental chamber, whose boundary hyperplanes are the reflecting hyperplanes of the reflections in $S$.

Let $\Delta:=\left\{\alpha_{s} \mid s \in S\right\}$ denote the simple roots and $\nabla:=\left\{\omega_{s} \mid s \in S\right\}$ denote the fundamental weights, defined such that $s\left(\omega_{t}\right)=\omega_{t}-\delta_{s=t} \alpha_{s}$ for all $s, t \in S$. In other words, $\Delta$ and $\nabla$ are dual bases of the Euclidean space $V$ (up to renormalization). Geometrically, simple roots and fundamental weights give respectively normal vectors and rays of the fundamental chamber $\mathcal{C}$. Let $\Phi:=\{w(\alpha) \mid w \in W, \alpha \in \Delta\}$ be the root system for $(W, S)$, with positive roots $\Phi^{+}:=\Phi \cap \mathbb{R}_{\geq 0} \Delta$ and negative roots $\Phi^{-}:=-\Phi^{+}$.

A reduced expression for an element $w \in W$ is a minimal length expression of $w$ as a product of generators in $S$. Let $w_{\text {。 }}$ denote the longest element of $W$, which sends the fundamental chamber $\mathcal{C}$ to its negative $-\mathcal{C}$. We let $\varphi: S \rightarrow S$ denote its conjugation action on the generators defined by $\varphi(s):=w_{\circ} s w_{\circ}$. Observe that $w_{\circ}\left(\alpha_{s}\right)=-\alpha_{\varphi(s)}$ and $w_{\circ}\left(\omega_{s}\right)=-\omega_{\varphi(s)}$.

We refer to [Hum90, Hum78] for further details on Coxeter groups.

Example 2.1 (Symmetric groups). The symmetric group $\mathfrak{S}_{n+1}$, acting on the hyperplane $H:=\left\{x \in \mathbb{R}^{n+1} \mid \sum x_{i}=0\right\}$ by permutation of the coordinates and generated by the adjacent transpositions $\tau_{p}:=(p p+1)$, is the type $A_{n}$ Coxeter system. Its simple roots are $\Delta:=\left\{e_{p+1}-e_{p} \mid p \in[n]\right\}$, its fundamental weights are $\nabla:=\left\{\sum_{q>p} e_{q} \mid p \in[n]\right\}$, and its root system is $\Phi:=\left\{e_{p}-e_{q} \mid p \neq q \in[n+1]\right\}$. Note that we have chosen the fundamental weights to match usual conventions, even if they do not live in the hyperplane $H$. The careful reader might prefer to project these weights down to $H$ and adapt our discussions below accordingly. Figures 1(a) and 2(a) show the type $A_{2}$ and $A_{3}$ Coxeter arrangements.

2.2. Permutahedra. Let $u$ be a point in the interior of the fundamental chamber. We write $u:=\sum_{s \in S} u_{s} \omega_{s}$ with $u_{s} \in \mathbb{R}_{>0}$. The $W$-permutahedron $\operatorname{Perm}^{u}(W)$ is the convex hull of the orbit of $u$ under $W$. Its combinatorial properties are determined by those of the Coxeter group $W$. Let us observe in particular that:

(i) The normal fan of $\operatorname{Perm}^{u}(W)$ is the Coxeter fan.

(ii) For any $w \in W$ and $s \in S$, the facet of $\operatorname{Perm}^{u}(W)$ orthogonal to $w\left(\omega_{s}\right)$ is defined by the inequality $\left\langle w\left(\alpha_{s}\right) \mid x\right\rangle \leq\left\langle\alpha_{s} \mid u\right\rangle$ and supported by the hyperplane $w\left(u+\operatorname{vect}\left(\Delta \backslash \alpha_{s}\right)\right)$.

(iii) Each face of $\operatorname{Perm}^{u}(W)$ is a $W^{\prime}$-permutahedron for a parabolic subgroup $W^{\prime}$ of $W$, and the faces of $\operatorname{Perm}^{u}(W)$ are naturally parametrized by cosets of parabolic subgroups of $W$.

We refer to the detailed survey on the $W$-permutahedra in Hoh12. 


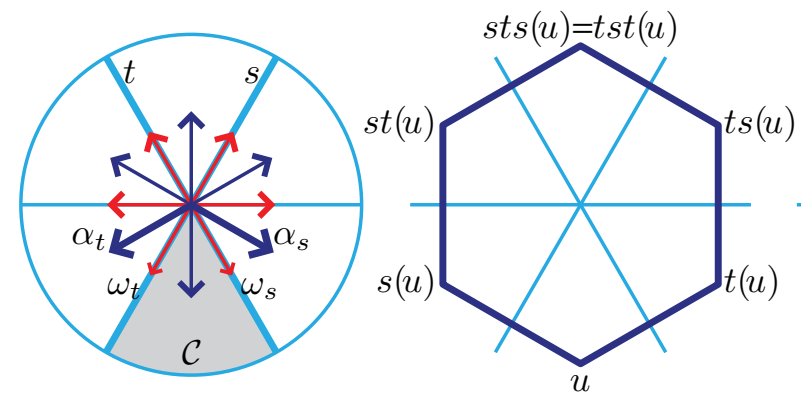

(a) (b)

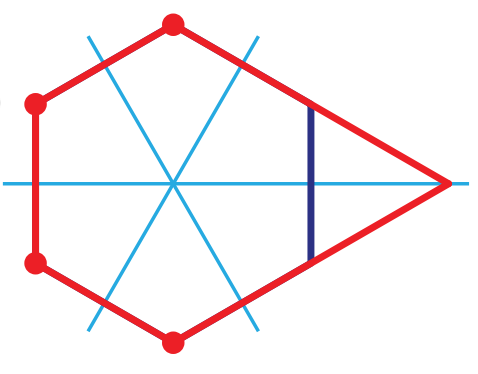

(c)

Figure 1. The type $A_{2}$ Coxeter arrangement (a) and the balanced $A_{2}$-permutahedron (b) and (st)-associahedron (c).

If $u=\mathbf{1}:=\sum_{\omega \in \nabla} \omega$, we say that the $W$-permutahedron $\operatorname{Perm}^{u}(W)$ is balanced, and we simply denote it by $\operatorname{Perm}(W)$ rather than $\operatorname{Perm}^{\mathbf{1}}(W)$. Note that the balanced $W$-permutahedron $\operatorname{Perm}(W)$ is (a translate of) the Minkowski sum of all positive roots (each considered as a one-dimensional polytope). Finally, we say that the $W$-permutahedron $\operatorname{Perm}(W)$ is fairly balanced if $w_{\circ}(u)=-u$, i.e. if $u_{s}=u_{\varphi(s)}$ for all $s \in S$.

Example 2.2 (Classical permutahedron). The classical permutahedron is the convex hull of all permutations of $\{0, \ldots, n\}$, regarded as vectors in $\mathbb{R}^{n+1}$. According to our choice of fundamental weights, we have $\sum_{\omega \in \nabla} \omega=(0, \ldots, n)$, so that the classical permutahedron coincides with the balanced $A_{n}$-permutahedron $\operatorname{Perm}\left(A_{n}\right)$. Figures 1(b) and 2(b) show balanced $A_{2^{-}}$and $A_{3}$-permutahedra.

2.3. Associahedra. We now recall the construction of generalized associahedra given by C. Hohlweg, C. Lange, and H. Thomas in [HLT11, based on the notions of sortable elements, Cambrian lattices and Cambrian fans defined and studied in Rea06, Rea07, RS09].

Fix a Coxeter element $c$ of $W$ and a reduced expression c of $c$. That is to say, c is a word on $S$ where each simple reflection appears precisely once. We say that $s \in S$ is initial in $c$ if there is a reduced expression for $c$ starting with $s$. For $w \in W$, we denote by $\mathrm{w}(\mathrm{c})$ the c-sorting word of $w$, i.e. the lexicographically first (as a sequence of positions) reduced subword of $\mathrm{c}^{\infty}$ for $w$. This word can be written as $\mathrm{w}(\mathrm{c})=\mathrm{c}_{K_{1}} \mathrm{c}_{K_{2}} \cdots \mathrm{c}_{K_{p}}$, where $\mathrm{c}_{K}$ denotes the subword of $\mathrm{c}$ taking only the simple reflections in $K \subset S$ into account. The element $w$ is then called c-sortable if $K_{1} \supseteq K_{2} \supseteq \cdots \supseteq K_{p}$. Observe that the property of being $c$-sortable does not depend on the particular reduced expression $\mathrm{c}$ of the Coxeter element $c$. Finally, $w$ is called $c$-singleton if $w$ is $c$-sortable and $w w_{\circ}$ is $\left(c^{-1}\right)$-sortable.

The $c$-associahedron $\operatorname{Asso}_{c}^{u}(W)$ is obtained from $\operatorname{Perm}^{u}(W)$ by removing all boundary hyperplanes not containing any vector $w(u)$ for a $c$-singleton $w$ of $W$. The boundary complex of its polar is the cluster complex defined by S. Fomin and A. Zelevinsky in FZ03a, and its normal fan is the c-Cambrian fan defined by N. Reading and D. Speyer RS09. Note that the combinatorics of the $c$ associahedron (the cluster complex) does not depends on $c$, while its geometry (in particular, the $c$-Cambrian fan) does. We denote by $\mathfrak{B}^{u}(c)$ the barycenter of 


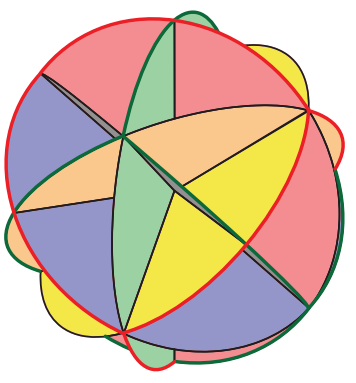

(a)

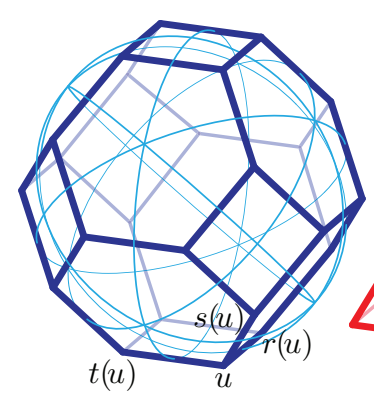

(b)



(c)

Figure 2. The type $A_{3}$ Coxeter arrangement (a) and the balanced $A_{3}$-permutahedron (b) and (rst)-associahedron (c).

the $c$-associahedron $\operatorname{Asso}_{c}^{u}(W)$. We say that $\operatorname{Asso}_{c}^{u}(W)$ is balanced or fairly balanced if $\operatorname{Perm}^{u}(W)$ is. We simply denote by $\operatorname{Asso}_{c}(W)$ and $\mathfrak{B}(c)$ the balanced $c$-associahedron and its barycenter.

Example 2.3 (Loday's associahedron). The cluster complex of type $A_{n}$ is isomorphic to the simplicial complex of crossing-free sets of internal diagonals of a convex $(n+3)$-gon. Its vertices are internal diagonals and its facets are triangulations of the $(n+3)$-gon. In Lod04, J.-L. Loday provided an elegant realization of this simplicial complex, based on the following vertex description. Label the vertices of the $(n+3)$-gon cyclically from 0 to $n+2$. Then, associate to each triangulation $T$ of the $(n+3)$-gon its Loday vector $\mathrm{L}(T)$ whose $j^{\text {th }}$ coordinate is given by:

$\mathrm{L}(T)_{j}:=(j-\min \{i \in[0, j-2] \mid i j \in T\}) \cdot(\max \{k \in[j+2, n+2] \mid j k \in T\}-j)$.

Loday's associahedron is the convex hull of the Loday vectors of all triangulations of the $(n+3)$-gon:

$$
\mathrm{L}_{n}:=\operatorname{conv}\{\mathrm{L}(T) \mid T \text { triangulation of the }(n+3) \text {-gon }\} \text {. }
$$

In fact, Loday's associahedron $\mathrm{L}_{n}$ coincides with the balanced $\left(\tau_{1} \cdots \tau_{n}\right)$-associahedron Asso $_{\tau_{1} \cdots \tau_{n}}\left(A_{n}\right)$. This polytope is illustrated in Figures 1(c) and 2(c) for types $A_{2}$ and $A_{3}$. Loday's vertex description of the associahedron was extended to a vertex description of all $c$-associahedra of type $A$ and $B$ in [HL07.

2.4. Brick polytopes. In the remainder of this section, we recall the viewpoint on $c$-associahedra coming from brick polytopes. We refer to [PS11] for the general treatment and to $[\mathrm{PS} 12$ for a specific treatment of the type $A$ situation.

2.4.1. Subword complexes. For a word $\mathrm{Q}:=\mathrm{q}_{1} \mathrm{q}_{2} \cdots \mathrm{q}_{m}$ on $S$ and an element $\rho \in W$, A. Knutson and E. Miller define in KM04 the subword complex $\mathcal{S C}(\mathrm{Q}, \rho)$ to be the simplicial complex of those subwords of $\mathrm{Q}$ whose complements contain a reduced expression for $\rho$ as a subword. A vertex of $\mathcal{S C}(\mathrm{Q}, \rho)$ is a position of a letter in $\mathrm{Q}$. We denote by $[m]:=\{1,2, \ldots, m\}$ the set of positions in Q. A facet of $\mathcal{S C}(\mathrm{Q}, \rho)$ is the complement of a set of positions which forms a reduced expression for $\rho$ in $\mathrm{Q}$.

In this paper, we consider only spherical subword complexes $\mathcal{S C}(\mathrm{Q}, \rho)$ for which we can assume that $\rho=w_{\circ}$ and Q contains a reduced expression for $w_{\circ}$ (see [CLS11, Theorem 3.7]). We write $\mathcal{S C}(\mathrm{Q})$ instead of $\mathcal{S C}\left(\mathrm{Q}, w_{\circ}\right)$ to shorten notation. 
To avoid confusion between the words on $S$ and the elements of $W$, we use roman letters like $\mathrm{w}:=\mathrm{w}_{1} \cdots \mathrm{w}_{p}$ for words and italic letters like $w=w_{1} \cdots w_{p}$ for group elements. The distinction is in general clear from the context and can usually be ignored.

Example 2.4. Consider the Coxeter group $\mathfrak{S}_{3}=\left\langle\tau_{1}, \tau_{2}\right\rangle=\langle s, t\rangle$ and the word $\mathrm{Q}_{2}:=$ ststs. The reduced expressions of $w_{\circ}$ are sts and tst. Therefore, the facets of $\mathcal{S C}\left(\mathrm{Q}_{2}\right)$ are $\{1,2\},\{2,3\},\{3,4\},\{4,5\}$, and $\{1,5\}$, and the subword complex $\mathcal{S C}\left(\mathrm{Q}_{2}\right)$ is a pentagon. Similarly, for Coxeter group $\mathfrak{S}_{4}=\left\langle\tau_{1}, \tau_{2}, \tau_{3}\right\rangle=\langle r, s, t\rangle$ and the word $\mathrm{Q}_{3}:=$ rstrstrsr, the subword complex $\mathcal{S C}\left(\mathrm{Q}_{3}\right)$ is isomorphic to the cluster complex of type $A_{3}$.

Example 2.5 (Primitive sorting networks). In type $A_{n}$, we can represent the word $\mathrm{Q}=\mathrm{q}_{1} \mathrm{q}_{2} \cdots \mathrm{q}_{m}$ by a sorting network $\mathcal{N}_{\mathrm{Q}}$ as illustrated in Figure 3 (left). The network $\mathcal{N}_{\mathrm{Q}}$ is formed by $n+1$ horizontal lines (its levels, labeled from bottom to top) together with $m$ vertical segments (its commutators, labeled from left to right) corresponding to the letters of $\mathrm{Q}$. If $\mathrm{q}_{k}=\tau_{p}$, the $k^{\text {th }}$ commutator of $\mathcal{N}_{\mathrm{Q}}$ lies between the $p^{\text {th }}$ and $(p+1)^{\text {th }}$ levels of $\mathcal{N}_{\mathrm{Q}}$.
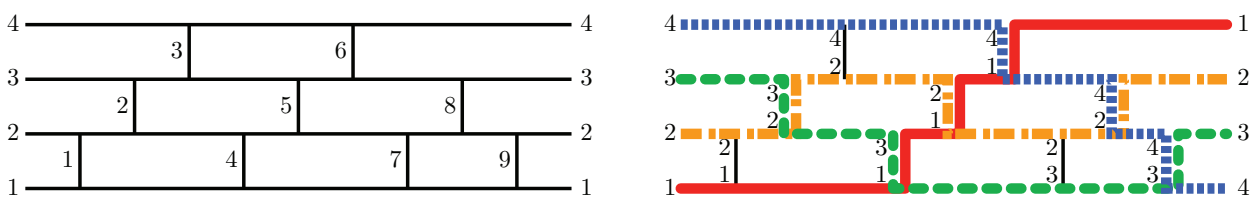

FIGURE 3 . The sorting network $\mathcal{N}_{\mathrm{Q}_{3}}$ and the pseudoline arrangement $\Lambda_{\{1,3,7\}}$.

A pseudoline supported by $\mathcal{N}_{\mathrm{Q}}$ is an abscissa monotone path on the network $\mathcal{N}_{\mathrm{Q}}$. A commutator of $\mathcal{N}_{\mathrm{Q}}$ is a crossing between two pseudolines if it is traversed by both pseudolines, and a contact if its endpoints are contained one in each pseudoline. A pseudoline arrangement $\Lambda$ (with contacts) is a set of $n+1$ pseudolines on $\mathcal{N}_{\mathrm{Q}}$, any two of which have precisely one crossing, possibly some contacts, and no other intersection. As a consequence of the definition, the pseudoline of $\Lambda$ which starts at level $p$ ends at level $n-p+2$ and is called the $p^{\text {th }}$ pseudoline of $\Lambda$. As illustrated in Figure 3 (right), a facet $I$ of $\mathcal{S C}(\mathrm{Q})$ is represented by a pseudoline arrangement $\Lambda_{I}$ supported by $\mathcal{N}_{\mathrm{Q}}$. Its contacts (resp. crossings) are the commutators of $\mathcal{N}_{\mathrm{Q}}$ corresponding to the letters of $I$ (resp. of the complement of $I$ ).

A brick of $\mathcal{N}_{\mathrm{Q}}$ is a connected component of its complement, bounded on the right by a commutator of $\mathcal{N}_{\mathrm{Q}}$. For $k \in[m]$, the $k^{\text {th }}$ brick is that immediately to the left of the $k^{\text {th }}$ commutator of $\mathcal{N}_{\mathrm{Q}}$.

2.4.2. Brick polytopes. In PS11, we constructed a polytope associated to a spherical subword complex $\mathcal{S C}(\mathrm{Q})$ as follows. To a facet $I$ of $\mathcal{S C}(\mathrm{Q})$ and a position $k$ in $\mathrm{Q}$, we associate a root and a weight

$$
\mathrm{r}_{\mathrm{Q}}(I, k):=\Pi \mathrm{Q}_{[k-1] \backslash I}\left(\alpha_{q_{k}}\right) \text { and } \mathrm{w}_{\mathrm{Q}}(I, k):=\Pi \mathrm{Q}_{[k-1] \backslash I}\left(\omega_{q_{k}}\right),
$$

where $\Pi_{X}$ denotes the product of the reflections $q_{x} \in \mathrm{Q}$ for $x \in X$. The brick 
vector of the facet $I$ is the vector

$$
\mathrm{B}_{\mathrm{Q}}(I):=\sum_{k \in[m]} \mathrm{w}_{\mathrm{Q}}(I, k),
$$

and the brick polytope $\mathcal{B}(\mathrm{Q})$ is the convex hull of all the brick vectors,

$$
\mathcal{B}(\mathrm{Q}):=\operatorname{conv}\left\{\mathrm{B}_{\mathrm{Q}}(I) \mid I \text { facet of } \mathcal{S C}(\mathrm{Q})\right\} .
$$

Example 2.6 (Counting bricks). In type $A$, the above definitions for $\mathrm{r}_{\mathrm{Q}}(I, k)$, $\mathrm{w}_{\mathrm{Q}}(I, k)$, and $\mathrm{B}_{\mathrm{Q}}(I)$ can be visually interpreted on the pseudoline arrangement $\Lambda_{I}$ defined in Example 2.5. Namely, for any facet $I$ of $\mathcal{S C}(\mathrm{Q})$, any position $k \in[m]$, and any $p \in[n+1]$,

(i) the root $\mathrm{r}_{\mathrm{Q}}(I, k)$ is the difference $e_{t}-e_{b}$, where $t$ and $b$ are such that the $t^{\text {th }}$ and $b^{\text {th }}$ pseudolines of $\Lambda_{I}$ arrive respectively on top and bottom of the $k^{\text {th }}$ commutator of $\mathcal{N}_{\mathrm{Q}}$;

(ii) the weight $\mathrm{w}_{\mathrm{Q}}(I, k)$ is the characteristic vector of the pseudolines of $\Lambda_{I}$ which pass above the $k^{\text {th }}$ brick of $\mathcal{N}_{\mathrm{Q}}$;

(iii) the $p^{\text {th }}$ coordinate of the brick vector $\mathrm{B}_{\mathrm{Q}}(I)$ is the number of bricks of $\mathcal{N}_{\mathrm{Q}}$ below the $p^{\text {th }}$ pseudoline of $\Lambda_{I}$.

We refer to PS12] for further details on type $A$ brick polytopes.

As observed in CLS11, Section 3.1], the root function $r_{Q}(\cdot, \cdot)$ encodes the combinatorics of flips in the subword complex $\mathcal{S C}(\mathrm{Q})$. We will mainly use here that $\mathrm{r}_{\mathrm{Q}}(I, \cdot)$ is a bijection between the complement of $I$ and $\Phi^{+}$, and thus that

$$
\sum_{k \notin I} \mathrm{r}_{\mathrm{Q}}(I, k)=\sum_{\beta \in \Phi^{+}} \beta .
$$

In [PS11, we proved that (the dual of) the brick polytope $\mathcal{B}(\mathrm{Q})$ realizes the subword complex if and only if the root configuration $\left\{\left\{\mathrm{r}_{\mathrm{Q}}(I, k) \mid i \in I\right\}\right\}$ of any (or equivalently all) facet(s) $I$ of $\mathcal{S C}(\mathrm{Q})$ is linearly independent. The following family is the main motivating example for the brick polytope construction.

2.4.3. Generalized associahedra and brick polytopes. Let $c$ be a Coxeter element of $W$, let $\mathrm{c}$ be a reduced expression for $c$, and let $\mathrm{w}_{\circ}(\mathrm{c}):=\mathrm{w}_{1} \cdots \mathrm{w}_{N}$ denote the c-sorting word for the longest element $w_{\circ}$. According to [CLS11], the subword complex $\mathcal{S C}_{\mathrm{c}}:=\mathcal{S C}\left(\mathrm{cw}_{\circ}(\mathrm{c})\right)$ is (isomorphic to) the cluster complex of type $W$. We furthermore proved in [PS11, Theorem 6.1] that the brick polytope $\mathcal{B}_{\mathrm{c}}:=\mathcal{B}\left(\mathrm{cw}_{\circ}(\mathrm{c})\right)$ is indeed a polytopal realization of $\mathcal{S C}_{\mathrm{c}}$. Finally, we also proved in PS11, Theorem 6.6] that - up to affine translation by a vector $\Omega_{c}$ - the brick polytope $\mathcal{B}_{\mathrm{c}}$ coincides with the balanced $c$-associahedron $\operatorname{Asso}_{c}(W)$. More explicitly, we have

$$
\operatorname{Asso}_{c}(W)=\operatorname{conv}\left\{\mathrm{B}_{\mathrm{c}}(I)-\Omega_{c} \mid I \text { facet of } \mathcal{S C}_{\mathrm{c}}\right\},
$$

where the affine translation $\Omega_{c}$ is given by

$$
\Omega_{c}:=\sum_{k \in[N]} w_{1} \cdots w_{k-1}\left(\omega_{w_{k}}\right)
$$

where $\mathrm{w}_{1} \ldots \mathrm{w}_{N}$ is the c-sorting word for $w_{\circ}$. In equality (2) and throughout the paper, we abuse notation and write $\mathrm{B}_{\mathrm{c}}(I)$ rather than $\mathrm{B}_{\mathrm{cw}_{\circ}(\mathrm{c})}(I)$, and similarly for $\mathrm{r}_{\mathrm{c}}(I, k)$ and $\mathrm{w}_{\mathrm{c}}(I, k)$, as we already $\operatorname{did}$ for $\mathcal{S C}_{\mathrm{c}}$ and for $\mathcal{B}_{\mathrm{c}}$. 
Example 2.7. The words $\mathrm{Q}_{2}$ and $\mathrm{Q}_{3}$ of Example 2.4 are precisely $(\mathrm{st}) \mathrm{w}_{\circ}(\mathrm{st})$ and (rst) $\mathrm{w}_{\mathrm{o}}$ (rst). Therefore, the brick polytopes $\mathcal{B}\left(\mathrm{Q}_{2}\right)=\mathcal{B}_{\text {st }}$ and $\mathcal{B}\left(\mathrm{Q}_{3}\right)=\mathcal{B}_{\text {rst }}$ coincide, up to translation, with Loday's associahedra from Figures 1 (c) and 2 (c).

More generally, label the vertices of the $(n+3)$-gon cyclically from 0 to $(n+2)$ and set $\mathrm{c}:=\tau_{1} \tau_{2} \cdots \tau_{n}$. Consider the map sending the $i^{\text {th }}$ letter of $\mathrm{cw}_{\circ}(\mathrm{c})$ to the $i^{\text {th }}$ internal diagonal of the $(n+3)$-gon in lexicographic order. This map induces an isomorphism between the simplicial complex of crossing free sets of internal diagonals of the $(n+3)$-gon and the type $A_{n}$ subword complex $\mathcal{S C}_{\mathrm{c}}$. See Woo04, Stu11, PP12 for details and extensions of this isomorphism. The brick polytope $\mathcal{B}_{\mathrm{c}}$ then coincides with Loday's associahedron $L_{n}$.

2.4.4. Affine translation and greedy facets. The positive greedy facet $\mathrm{P}(\mathrm{Q})$ (resp. the negative greedy facet $\mathrm{N}(\mathrm{Q})$ ) is the lexicographically first (resp. last) facet of $\mathcal{S C}(\mathrm{Q})$. It turns out that $\mathrm{P}(\mathrm{Q})$ (resp. $\mathrm{N}(\mathrm{Q})$ ) is the unique facet whose root configuration $\left\{\left\{\mathrm{r}_{\mathrm{Q}}(I, k) \mid i \in I\right\}\right\}$ contains only positive (resp. negative) roots. These two particular facets were defined and studied in PS13. to construct EL-labelings and canonical spanning trees for subword complexes.

We now focus on the situation of $\mathcal{S C}_{c}$. To simplify notation, we set here as well $\mathrm{P}_{\mathrm{c}}:=\mathrm{P}\left(\mathrm{cw}_{\mathrm{o}}(\mathrm{c})\right)$ and $\mathrm{N}_{\mathrm{c}}:=\mathrm{N}\left(\mathrm{cw}_{\mathrm{o}}(\mathrm{c})\right)$. Observe that $\mathrm{P}_{\mathrm{c}}$ is the set of positions of the first appearance of the generators of $S$ within $\mathrm{cw}_{\circ}(\mathrm{c})$. Similarly, $\mathrm{N}_{\mathrm{c}}$ is the set of positions of the last appearance in $\mathrm{cw}_{\circ}(\mathrm{c})$ of the generators of $S$. Up to transpositions of consecutive commuting letters, these are moreover the first and the last $n$ positions in $\mathrm{cw}_{\circ}(\mathrm{c})$.

In equality (2), the vertices of $\mathcal{B}_{c}-\Omega_{c}$ corresponding to the positive and negative greedy facets $\mathrm{P}_{\mathrm{c}}$ and $\mathrm{N}_{\mathrm{c}}$ coincide respectively with the vertices $e(\mathbf{1})=\mathbf{1}$ and $w_{\circ}(\mathbf{1})=-\mathbf{1}$ of $\operatorname{Asso}_{c}(W)$. This implies that

$$
\Omega_{c}=\mathrm{B}_{\mathrm{c}}\left(\mathrm{P}_{\mathrm{c}}\right)-\mathbf{1}=\mathrm{B}_{\mathrm{c}}\left(\mathrm{N}_{\mathrm{c}}\right)+\mathbf{1} .
$$

2.4.5. The brick polytope for arbitrary basepoints. We finally describe the situation of a general basepoint $u:=\sum_{s \in S} u_{s} \omega_{s}$ in the interior of the fundamental chamber. As observed in [PS11, Remark 6.11], the brick polytope construction and its realization properties remain valid if we replace the root and weight functions by

$$
\mathrm{r}_{\mathrm{Q}}^{u}(I, k):=u_{q_{k}} \mathrm{r}_{\mathrm{Q}}(I, k) \text { and } \mathrm{w}_{\mathrm{Q}}^{u}(I, k):=u_{q_{k}} \mathrm{w}_{\mathrm{Q}}(I, k),
$$

the brick vector by

$$
\mathrm{B}_{\mathrm{Q}}^{u}(I):=\sum_{k \in[m]} \mathrm{w}_{\mathrm{Q}}^{u}(I, k)=\sum_{k \in[m]} u_{q_{k}} \mathrm{w}_{\mathrm{Q}}(I, k),
$$

and the brick polytope by

$$
\mathcal{B}^{u}(\mathrm{Q}):=\operatorname{conv}\left\{\mathrm{B}_{\mathrm{Q}}^{u}(I) \mid I \text { facet of } \mathcal{S C}(\mathrm{Q})\right\} .
$$

The polytope $\mathcal{B}^{u}(\mathrm{Q})$ is a deformation of $\mathcal{B}(\mathrm{Q})$. Its combinatorics and its normal fan are controlled by the subword complex $\mathcal{S C}(\mathrm{Q})$, and therefore are independent of the basepoint $u$, but its geometry (for example its edge lengths) is determined by $u$.

For the subword complex $\mathcal{S C}_{\mathrm{c}}$, the polytope $\mathcal{B}_{c}^{u}$ is a translate of the $c$-associahedron $\operatorname{Asso}_{c}^{u}(W)$. More precisely,

$$
\operatorname{Asso}_{c}^{u}(W)=\operatorname{conv}\left\{\mathrm{B}_{\mathrm{c}}^{u}(I)-\Omega_{c}^{u} \mid I \text { facet of } \mathcal{S C}_{\mathrm{c}}\right\},
$$


where the affine translation $\Omega_{c}^{u}$ is now given by

$$
\Omega_{c}^{u}:=\mathrm{B}_{\mathrm{c}}^{u}\left(\mathrm{P}_{\mathrm{c}}\right)-u=\sum_{k \in[N]} u_{q_{k}} w_{1} \cdots w_{k-1}\left(\omega_{w_{k}}\right) .
$$

\section{THE PROOF}

We first focus on balanced associahedra and discuss the extension to fairly balanced associahedra in Section 3.3. In Section 3.4, we will discuss a further orbit refinement. In view of (2), the balanced version of Theorem 1.1 reduces to the following theorem.

Theorem 3.1. The vertex barycenter of the translated brick polytope $\mathcal{B}_{c}-\Omega_{c}$ coincides with that of the balanced $W$-permutahedron $\operatorname{Perm}(W)$. That is,

$$
\sum\left(\mathrm{B}_{\mathrm{c}}(I)-\Omega_{c}\right)=0,
$$

where the sum ranges over all facets $I$ of $\mathcal{S C}_{\mathrm{c}}$.

The proof of Theorem 3.1 has two steps:

(i) We first prove that the barycenters of all balanced $c$-associahedra $\operatorname{Asso}_{c}(W)$ coincide, i.e. that $\mathfrak{B}(c)=\mathfrak{B}\left(c^{\prime}\right)$ for any Coxeter elements $c, c^{\prime}$.

(ii) We then prove that the barycenter of the superposition of the vertex set of $\operatorname{Asso}_{c}(W)$ with the vertex set of $\operatorname{Asso}_{c^{-1}}(W)$ coincides with the origin, i.e. that $\mathfrak{B}(c)+\mathfrak{B}\left(c^{-1}\right)=0$.

We will deduce these two statements from Lemmas 3.2 [3.3 3.4 and 3.5, which describe the impact on brick vectors of four natural operations on the word Q.

3.1. Four operations. In the next four lemmas, we study the behavior of the brick vectors of the facets of $\mathcal{S C}(\mathrm{Q})$ under four natural operations on the word $\mathrm{Q}$, namely when we commute, rotate, conjugate, or reverse Q. The first two operations were as well considered in CLS11, Propositions 3.8 and 3.9]. Although we will use them later only for words of the form $\mathrm{cw}_{\circ}(\mathrm{c})$, the statements below are valid for any word Q.

Lemma 3.2 (Commute). If $\pi(\mathrm{Q}):=\mathrm{q}_{\pi(1)} \cdots \mathrm{q}_{\pi(m)}$ is obtained from $\mathrm{Q}=\mathrm{q}_{1} \cdots \mathrm{q}_{m}$ by a sequence of transpositions of consecutive commuting letters, then $\pi$ induces an isomorphism between the subword complexes $\mathcal{S C}(\mathrm{Q})$ and $\mathcal{S C}(\pi(\mathrm{Q}))$. Moreover,

$$
\mathrm{B}_{\pi(\mathrm{Q})}(\pi(I))=\mathrm{B}_{\mathrm{Q}}(I)
$$

for any facet $I$ of $\mathcal{S C}(\mathrm{Q})$.

Proof. The isomorphism is obtained directly from the definition of subword complexes; see [CLS11, Proposition 3.8]. Moreover, the definition of the weight function implies that

$$
\mathrm{w}_{\pi(\mathrm{Q})}(\pi(I), \pi(k))=\mathrm{w}_{\mathrm{Q}}(I, k)
$$

for any facet $I$ and position $k$. The result follows by summation.

Lemma 3.3 (Rotate). Let $\mathrm{Q}^{\circlearrowleft}:=\mathrm{q}_{2} \cdots \mathrm{q}_{m} \varphi\left(\mathrm{q}_{1}\right)$ be the rotation of $\mathrm{Q}=\mathrm{q}_{1} \cdots \mathrm{q}_{m}$. Then the cyclic rotation $\rho: i \mapsto(i-1)$, where we identify 0 and $m$, induces an isomorphism between the subword complexes $\mathcal{S C}(\mathrm{Q})$ and $\mathcal{S C}\left(\mathrm{Q}^{\circlearrowleft}\right)$. Moreover,

$$
\mathrm{B}_{\mathrm{Q} \circlearrowleft}(\rho(I))-\mathrm{B}_{\mathrm{Q}}(I) \in-2 \omega_{q_{1}}+\mathbb{R} \alpha_{q_{1}}
$$

for any facet I of $\mathcal{S C}(\mathrm{Q})$. 
Proof. The isomorphism is again obtained directly from the definition of subword complexes; see CLS11, Proposition 3.9]. From the definition of the weight function, we furthermore obtain that for any facet $I$ of $\mathcal{S C}(\mathrm{Q})$ and position $k>1$,

$$
\mathrm{w}_{\mathrm{Q}^{\circlearrowleft}}(\rho(I), \rho(k))= \begin{cases}\mathrm{w}_{\mathrm{Q}}(I, k) & \text { if } 1 \in I, \\ q_{1}\left(\mathrm{w}_{\mathrm{Q}}(I, k)\right) & \text { if } 1 \notin I,\end{cases}
$$

and moreover,

$$
\mathrm{w}_{\mathrm{Q}}(\rho(I), \rho(1))=\left\{\begin{aligned}
w_{\circ}\left(\omega_{\varphi\left(q_{1}\right)}\right) & =\mathrm{w}_{\mathrm{Q}}(I, 1)-2 \omega_{q_{1}} & & \text { if } 1 \in I, \\
w_{\circ} \cdot \varphi\left(q_{1}\right)\left(\omega_{\varphi\left(q_{1}\right)}\right) & =\mathrm{w}_{\mathrm{Q}}(I, 1)-2 \omega_{q_{1}}+\alpha_{q_{1}} & & \text { if } 1 \notin I .
\end{aligned}\right.
$$

The result follows by summation.

Lemma 3.4 (Conjugate). Let $\mathrm{Q}^{\uparrow}:=\varphi\left(q_{1}\right) \varphi\left(q_{2}\right) \ldots \varphi\left(q_{m}\right)$ be the $w_{\circ}$-conjugate of $\mathrm{Q}=\mathrm{q}_{1} \ldots \mathrm{q}_{m}$. Then the subword complexes $\mathcal{S C}(\mathrm{Q})$ and $\mathcal{S C}\left(\mathrm{Q}^{\uparrow}\right)$ coincide. Moreover,

$$
\mathrm{B}_{\mathrm{Q} \uparrow}(I)=-w_{\circ}\left(\mathrm{B}_{\mathrm{Q}}(I)\right)
$$

for any facet $I$ of $\mathcal{S C}(\mathrm{Q})$.

Proof. For any $I \subset[m]$, we have $\Pi \mathrm{Q}_{[m] \backslash I}^{\uparrow}=w_{\circ} \cdot \Pi \mathrm{Q}_{[m] \backslash I} \cdot w_{\circ}$, which ensures that $\mathcal{S C}(\mathrm{Q})=\mathcal{S C}\left(\mathrm{Q}^{\uparrow}\right)$. Remembering that $w_{\circ}\left(\omega_{s}\right)=-\omega_{\varphi(s)}$, a direct calculation from the definition of the weight function gives

$$
\begin{aligned}
\mathrm{w}_{\mathrm{Q}^{£}}(I, k) & =w_{\circ} \cdot \Pi \mathrm{Q}_{[k-1] \backslash I} \cdot w_{\circ}\left(\omega_{\varphi\left(q_{k}\right)}\right) \\
& =-w_{\circ} \cdot \Pi \mathrm{Q}_{[k-1] \backslash I}\left(\omega_{q_{k}}\right) \\
& =-w_{\circ}\left(\mathrm{w}_{\mathrm{Q}}(I, k)\right),
\end{aligned}
$$

for any facet $I$ of $\mathcal{S C}(\mathrm{Q})$ and any position $k$. The result follows by summation.

Lemma 3.5 (Reverse). Let $\mathrm{Q}^{\leftrightarrow}:=\mathrm{q}_{m} \cdots \mathrm{q}_{1}$ be the reverse of $\mathrm{Q}=\mathrm{q}_{1} \cdots \mathrm{q}_{m}$. Then the mirror $\mu: i \mapsto m-i+1$ induces an isomorphism between the subword complexes $\mathcal{S C}(\mathrm{Q})$ and $\mathcal{S C}(\mathrm{Q} \leftrightarrow)$. Moreover,

$$
\mathrm{B}_{\mathrm{Q}^{\leftrightarrow}}(\mu(I))=w_{\circ}\left(\mathrm{B}_{\mathrm{Q}}(I)\right)+\sum_{\beta \in \Phi^{+}} \beta
$$

for any facet I of $\mathcal{S C}(\mathrm{Q})$.

Proof. For any $I \subset[m]$, we have $\Pi \mathrm{Q}_{[m] \backslash \mu(I)}^{\overleftrightarrow{ }}=\left(\Pi \mathrm{Q}_{[m] \backslash I}\right)^{-1}$, which ensures that $\mu$ is an isomorphism between the subword complexes $\mathcal{S C}(\mathrm{Q})$ and $\mathcal{S C}(\mathrm{Q} \leftrightarrow)$. Consider now a facet $I$ of $\mathcal{S C}(\mathrm{Q})$. Since its complement in $\mathrm{Q}$ forms a reduced expression for $w_{\circ}$, we have

$$
w_{\circ}=\Pi \mathrm{Q}_{[m] \backslash I}= \begin{cases}\Pi \mathrm{Q}_{[k-1] \backslash I} \cdot \Pi \mathrm{Q}_{[k+1, m] \backslash I} & \text { if } k \in I, \\ \Pi \mathrm{Q}_{[k-1] \backslash I} \cdot q_{k} \cdot \Pi \mathrm{Q}_{[k+1, m] \backslash I} & \text { if } k \notin I .\end{cases}
$$

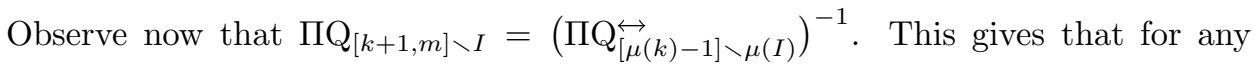
position $k \in I$, we have

$$
\begin{aligned}
\mathrm{w}_{\mathrm{Q}} \leftrightarrow(\mu(I), \mu(k)) & =\Pi \mathrm{Q}_{[\mu(k)-1] \backslash \mu(I)}^{\leftrightarrow}\left(\omega_{q_{k}}\right) \\
& =w_{\circ} \cdot \Pi \mathrm{Q}_{[k-1] \backslash I}\left(\omega_{q_{k}}\right) \\
& =w_{\circ}\left(\mathrm{w}_{\mathrm{Q}}(I, k)\right),
\end{aligned}
$$


and for any position $k \in[m] \backslash I$, we have

$$
\begin{aligned}
\mathrm{w}_{\mathrm{Q} \leftrightarrow}(\mu(I), \mu(k)) & =\Pi \mathrm{Q}_{[\mu(k)-1] \backslash \mu(I)}^{\leftrightarrow}\left(\omega_{q_{k}}\right) \\
& =w_{\circ} \cdot \Pi \mathrm{Q}_{[k-1] \backslash I} \cdot q_{k}\left(\omega_{q_{k}}\right) \\
& =w_{\circ} \cdot \Pi \mathrm{Q}_{[k-1] \backslash I}\left(\omega_{q_{k}}\right)-w_{\circ} \cdot \Pi \mathrm{Q}_{[k-1] \backslash I}\left(\alpha_{q_{k}}\right) \\
& =w_{\circ}\left(\mathrm{w}_{\mathrm{Q}}(I, k)\right)-w_{\circ}\left(\mathrm{r}_{\mathrm{Q}}(I, k)\right) .
\end{aligned}
$$

Since $\sum_{k \notin I} \mathrm{r}_{\mathrm{Q}}(I, k)=\sum_{\beta \in \Phi^{+}} \beta$ (as we have seen in equality (10), and since $-w_{\circ}$ fixes $\sum_{\beta \in \Phi^{+}} \beta$, the result follows by summation.

Combining Lemmas 3.4 and 3.5 , we obtain the following corollary.

Corollary 3.6. Denote by $\mathrm{Q}^{\lfloor\downarrow}:=\varphi\left(\mathrm{q}_{m}\right) \cdots \varphi\left(\mathrm{q}_{1}\right)$ the reverse and $w_{\circ}$-conjugate of $\mathrm{Q}=\mathrm{q}_{1} \cdots \mathrm{q}_{m}$. The mirror $\mu: i \mapsto m-i+1$ defines an isomorphism between the subword complexes $\mathcal{S C}(\mathrm{Q})$ and $\mathcal{S C}\left(\mathrm{Q}^{\star \uparrow}\right)$, and moreover

$$
\mathrm{B}_{\mathrm{Q}}(I)+\mathrm{B}_{\mathrm{Q}^{\uparrow} \uparrow}(\mu(I))=\sum_{\beta \in \Phi^{+}} \beta
$$

for any facet I of $\mathcal{S C}(\mathrm{Q})$.

Example 3.7. The results of this section can be visually interpreted in type $A$, using the sorting network interpretation of Example 2.5. Namely, fix a word Q on the generators $\left\{\tau_{1}, \ldots, \tau_{n}\right\}$ of the type $A_{n}$ Coxeter group and a facet $I$ of $\mathcal{S C}(\mathrm{Q})$. Remember from Example 2.6 that the $p^{\text {th }}$ coordinate of the brick vector $\mathrm{B}_{\mathrm{Q}}(I)$ counts the number of bricks below the $p^{\text {th }}$ pseudoline of $\Lambda_{I}$. We have the following relations between the sorting networks $\mathcal{N}_{\mathrm{Q}}, \mathcal{N}_{\mathrm{Q}^{\circlearrowleft}}, \mathcal{N}_{\mathrm{Q}^{\uparrow}}$, and $\mathcal{N}_{\mathrm{Q}^{\leftrightarrow}}$.

(i) The sorting network $\mathcal{N}_{\mathrm{Q}}$ is obtained from the sorting network $\mathcal{N}_{\mathrm{Q}}$ rotating its first commutator to the end. The pseudolines of $\Lambda_{\rho(I)}$ coincide with that of $\Lambda_{I}$, except the two pseudolines incident to the rotated commutator. Therefore, the brick vector $\mathrm{B}_{\mathrm{Q}}(I)$ also counts bricks of $\mathcal{N}_{\mathrm{Q}}$ below the pseudolines of $\Lambda_{I}$. The only difference concerns the contribution of the rotated brick. This corresponds to Lemma 3.3 .

(ii) The sorting network $\mathcal{N}_{\mathrm{Q} \uparrow}$ is obtained from $\mathcal{N}_{\mathrm{Q}}$ by a reflection with respect to the horizontal axis. Therefore, the brick vector $\mathrm{B}_{\mathrm{Q} \mathfrak{}}(I)$ also counts bricks of $\mathcal{N}_{\mathrm{Q}}$ above the pseudolines of $\Lambda_{I}$. This corresponds to Lemma 3.4.

(iii) The sorting network $\mathcal{N}_{\mathrm{Q}} \leftrightarrow$ is obtained from $\mathcal{N}_{\mathrm{Q}}$ by a reflection with respect to the vertical axis. Therefore, the brick vector $\mathrm{B}_{\mathrm{Q}} \leftrightarrow(I)$ also counts bricks of $\mathcal{N}_{\mathrm{Q}}$ below the pseudolines of $\Lambda_{I}$. The only difference is that $\mathrm{B}_{\mathrm{Q}}(I)$ counts the leftmost unbounded bricks and not the rightmost unbounded bricks, while $\mathrm{B}_{\mathrm{Q}} \leftrightarrow(I)$ does the contrary. This corresponds to Lemma 3.5.

3.2. Barycenter of balanced associahedra. With the four preliminary lemmas of the previous section, we are now ready to prove Theorem [3.1. As indicated earlier, we split the proof into two steps: we first prove that the barycenter $\mathfrak{B}(c)$ of the $c$-associahedron $\operatorname{Asso}_{c}(W)$ is independent of the Coxeter element $c$, and then that $\mathfrak{B}(c)+\mathfrak{B}\left(c^{-1}\right)=0$.

Proposition 3.8. All balanced c-associahedra $\operatorname{Asso}_{c}(W)$ have the same vertex barycenter; i.e. $\mathfrak{B}(c)=\mathfrak{B}\left(c^{\prime}\right)$ for any Coxeter elements $c, c^{\prime}$ of $W$. 
Proof. Fix a Coxeter element $c$ and a reduced expression $c:=c_{1} \cdots c_{n}$ of $c$. Let $c^{\prime}$ denote the Coxeter element with reduced expression $c^{\prime}:=c_{2} \cdots c_{n} c_{1}$ obtained from $c$ rotating its first letter. Up to commutations of consecutive commuting letters, the word $c^{\prime} w_{\circ}\left(c^{\prime}\right)$ coincides with the word $\mathrm{cw}_{\circ}(\mathrm{c})^{\circlearrowleft}$; see [CLS11, Proposition 4.3]. Let $\psi$ denote the resulting isomorphism between $\mathcal{S C}_{\mathrm{c}}$ and $\mathcal{S C}_{c^{\prime}}$. Lemmas 3.2 and 3.3 ensure that

$$
\mathrm{B}_{\mathrm{c}^{\prime}}(\psi(I))-\mathrm{B}_{\mathrm{c}}(I) \in-2 \omega_{c_{1}}+\mathbb{R} \alpha_{c_{1}}
$$

for any facet $I$ of $\mathcal{S C}_{\mathrm{c}}$. Applying this to the positive greedy facet $\mathrm{P}_{\mathrm{c}}$ and using equality (3), we moreover obtain that

$$
\Omega_{c^{\prime}}-\Omega_{c} \in-2 \omega_{c_{1}}+\mathbb{R} \alpha_{c_{1}} .
$$

Therefore, $\left(\mathrm{B}_{\mathrm{c}^{\prime}}(\psi(I))-\Omega_{c^{\prime}}\right)-\left(\mathrm{B}_{\mathrm{c}}(I)-\Omega_{c}\right) \in \mathbb{R} \alpha_{c_{1}}$ for any facet $I$ of $\mathcal{S C}_{\mathrm{c}}$. This translates on the barycenters to

$$
\mathfrak{B}\left(c^{\prime}\right)-\mathfrak{B}(c) \in \mathbb{R} \alpha_{c_{1}} .
$$

Consider now the sequence $c^{(0)}, c^{(1)}, \ldots, c^{(n)}$ of Coxeter elements obtained from $c$ by repeatedly rotating the first letter. That is to say, $c^{(0)}:=c=c_{1} \cdots c_{n}$, then $c^{(1)}:=c^{\prime}=c_{2} \cdots c_{n} c_{1}$, and in general $c^{(k)}:=c_{k+1} \cdots c_{n} c_{1} \cdots c_{k}$. Since $c^{(0)}=c^{(n)}$, we have

$$
\mathfrak{B}\left(c^{(1)}\right)-\mathfrak{B}\left(c^{(0)}\right)=\mathfrak{B}\left(c^{(1)}\right)-\mathfrak{B}\left(c^{(n)}\right)=\sum_{i \in[n-1]} \mathfrak{B}\left(c^{(i)}\right)-\mathfrak{B}\left(c^{(i+1)}\right) .
$$

According to equality (8), the left-hand side belongs to the line $\mathbb{R} \alpha_{c_{1}}$, while the right-hand side belongs to the vector space generated by $\left\{\alpha_{c_{2}}, \ldots, \alpha_{c_{n}}\right\}=\Delta \backslash \alpha_{c_{1}}$. Since $\Delta$ is a linear basis of $V$, this ensures that $\mathfrak{B}\left(c^{\prime}\right)=\mathfrak{B}(c)$, i.e. that the barycenter is preserved by the rotation of the first letter. Applying Lemma 3.2, this remains true for the rotation of any initial letter in $c$. Since all Coxeter elements are related by repeated rotations of initial letters, the statement follows.

Proposition 3.9. The barycenter of the superposition of the vertices of the two associahedra $\operatorname{Asso}_{c}(W)$ and $\mathrm{Asso}_{c^{-1}}(W)$ is the origin; i.e. $\mathfrak{B}(c)+\mathfrak{B}\left(c^{-1}\right)=0$ for any Coxeter element $c$.

Proof. Abusing notation, we write here $\mathrm{c}^{-1}$ for the reduced expression $\mathrm{c} \leftrightarrow$ of $c^{-1}$. As observed in [CLS11, Remark 6.6], the $\left(\mathrm{c}^{-1}\right)$-sorting word of $w_{\mathrm{o}}$ is, up to transpositions of consecutive commuting letters, obtained by reversing and conjugating the $c$-sorting word of $w_{\circ}$. Denote by $\psi$ the resulting isomorphism between $\mathcal{S C}_{\mathrm{c}}$ and $\mathcal{S C}_{c^{-1}}$. From Lemma 3.2 and Corollary 3.6, we obtain that

$$
\mathrm{B}_{\mathrm{c}}(I)+\mathrm{B}_{\mathrm{c}^{-1}}(\psi(I))=\sum_{\beta \in \Phi^{+}} \beta,
$$

for any facet $I$ of $\mathcal{S C}_{\mathrm{c}}$. Observe now that $\psi$ sends the positive greedy facet $\mathrm{P}_{\mathrm{c}}$ to the negative greedy facet $N_{c^{-1}}$. Using equality (3), the previous equality for $P_{c}$ thus yields

$$
\Omega_{c}+\Omega_{c^{-1}}=\sum_{\beta \in \Phi^{+}} \beta .
$$

Thus, $\left(\mathrm{B}_{\mathrm{c}}(I)-\Omega_{c}\right)+\left(\mathrm{B}_{\mathrm{c}^{-1}}(\psi(I))-\Omega_{c^{-1}}\right)=0$. This translates on the barycenters to

$$
\mathfrak{B}(c)+\mathfrak{B}\left(c^{-1}\right)=0,
$$

which concludes the proof. 
Proof of Theorem 3.1. For any Coxeter element $c$ of $W$, we obtain from Propositions 3.8 and 3.9 that $2 \mathfrak{B}(c)=\mathfrak{B}(c)+\mathfrak{B}\left(c^{-1}\right)=0$.

3.3. Barycenter of fairly balanced associahedra. By slight modifications of the arguments in the previous two sections, we are now ready to prove Theorem 1.1 in full generality.

Proof of Theorem 1.1. Let $u$ be a basepoint within the fundamental chamber $\mathcal{C}$ for which $w_{\circ}(u)=-u$; i.e. $u_{s}=u_{\varphi(s)}$ for all $s \in S$. By a careful analysis of the proofs of the four Lemmas 3.2 3.5 we can see that they all remain valid in slightly modified forms. Lemma 3.2 stays valid as it is. In Lemma 3.3, equality (4) in its proof implies that the summand $-2 \omega_{q_{1}}$ must be replaced by $-2 u_{q_{1}} \omega_{q_{1}}$. Lemma 3.4 stays valid, though we use in equality (5) that $u_{q_{k}}=u_{\varphi\left(q_{k}\right)}$. Lemma 3.5 stays valid as well, where $u_{q_{k}}=u_{\varphi\left(q_{k}\right)}$ is used in equalities (6) and (7). Using this, we finally obtain that Propositions 3.8 and 3.9 hold as well in the case of fairly balanced associahedra. This completes the proof of Theorem 1.1 in full generality.

3.4. Orbit barycenter. In Problem 3.4 in Hoh12 and the preceding discussion, C. Hohlweg remarks that the vertex barycenter construction in HLR10] depends on the fact that the rotation and reflection symmetries of the underlying convex polygon do not change the barycenter of the associahedron in type $A$. As we have seen above, Proposition 3.8 and Proposition 3.9 play analogous roles in the case of general finite Coxeter systems. Denote by shift $\mathrm{c}_{\mathrm{c}}$ the bijection of the facets of $\mathcal{S C}_{\mathrm{c}}$ induced by the rotation of all letters in c. In [CLS11, Theorem 8.10] it is shown that in type $A_{n}$ with $\mathrm{c}=\tau_{1} \cdots \tau_{n}$ being the long cycle, the operation shift corresponds to the cyclic rotation of the underlying $(n+3)$-gon. Denote moreover by $\psi_{1}$ an isomorphism between $\mathcal{S C}_{\mathrm{c}}$ and $\mathcal{S C}_{c^{-1}}$ obtained by rotation as in the proof of Proposition 3.8, and by $\psi_{2}$ an isomorphism between $\mathcal{S C}_{\mathrm{c}}$ and $\mathcal{S C}_{c^{-1}}$ obtained by reversing and $w_{\circ}$-conjugation as in the proof of Proposition 3.9. Observe that again in type $A_{n}$ with $\mathrm{c}=\tau_{1} \cdots \tau_{n}$, the operation $\psi_{2}^{-1} \circ \psi_{1}$ corresponds to a reflection of the $(n+3)$-gon. For all finite Coxeter systems, we obtain the following theorem.

Theorem 3.10. Let $\mathcal{O}$ be the shift ${ }_{\mathrm{c}}$-orbit of a facet $I$ of $\mathcal{S C}_{\mathrm{c}}$, and let $\mathcal{O}^{\prime}$ be the shift $_{\mathrm{c}}$-orbit of the facet $\left(\psi_{2}^{-1} \circ \psi_{1}\right)(I)$ of $\mathcal{S C}_{\mathrm{c}}$. Then

$$
\sum\left(\mathrm{B}_{\mathrm{c}}(I)-\Omega_{c}\right)=0 \text {, }
$$

where the sum ranges over the orbit $\mathcal{O}$ if the two orbits $\mathcal{O}$ and $\mathcal{O}^{\prime}$ are equal or over the disjoint union $\mathcal{O} \sqcup \mathcal{O}^{\prime}$ if they are different.

Proof. Observe that, as in type $A_{n-1}$, we have that shift $\mathrm{c}^{-1} \circ \psi_{1}=\psi_{1} \circ$ shift $_{\mathrm{c}}$, and as well shift $\mathrm{c}^{-1} \circ \psi_{2}=\psi_{2} \circ$ shift $_{\mathrm{c}}$. The statement then follows directly from Propositions 3.8 and 3.9 .

Example 3.11. We consider again the subword complex $\mathcal{S C}_{\mathrm{c}}=\mathcal{S C}$ (rstrstrsr) as in Example 2.4. We have seen in Example 2.7 that the map sending the $i^{\text {th }}$ letter of $\mathrm{cw}_{\circ}(\mathrm{c})$ to the $i^{\text {th }}$ internal diagonal of the $(n+3)$-gon in lexicographic order induces an isomorphism between the simplicial complex of crossing free sets of internal diagonals of the $(n+3)$-gon and the type $A_{n}$ subword complex. The 14 triangulations of the 6-gon are shown in Figure 4, and the facets of $\mathcal{S C}_{\mathrm{c}}$ corresponding to the first triangulations in each of the rotation orbits $\mathrm{A}-\mathrm{D}$ are given by

$$
\{1,2,3\}, \quad\{6,7,8\}, \quad\{5,6,7\}, \quad\{1,3,7\} .
$$



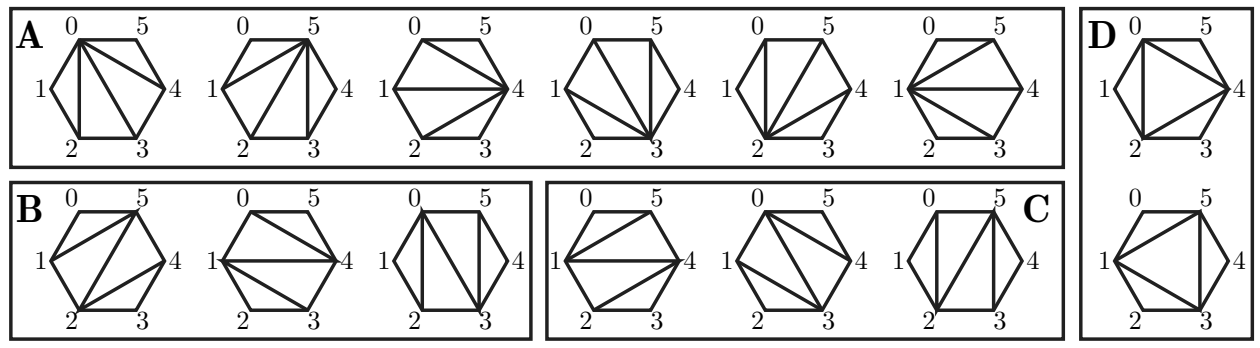

FIGURE 4. The four orbits under rotation of the 14 triangulations of the hexagon.

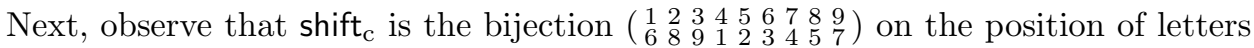
in $\mathrm{Q}_{3}$. It thus sends those 4 facets in (9) to the facets

$$
\{6,8,9\}, \quad\{3,4,5\}, \quad\{2,3,4\}, \quad\{4,6,9\},
$$

which exactly correspond to the second triangulation in each orbit. Finally, observe that $\psi_{2}^{-1} \circ \psi_{1}$ is the bijection $\left(\begin{array}{lllllllll}1 & 2 & 3 & 4 & 5 & 6 & 7 & 8 & 9 \\ 4 & 2 & 9 & 1 & 8 & 7 & 6 & 5 & 3\end{array}\right)$ and sends the 4 facets in (9) to the facets

$$
\{2,4,9\}, \quad\{5,6,7\}, \quad\{6,7,8\}, \quad\{4,6,9\} .
$$

This gives that orbit $\mathrm{A}$ is mapped onto itself, orbits $\mathrm{B}$ and $\mathrm{C}$ are interchanged, and orbit D is again mapped onto itself, as desired. Therefore, the barycenter of the vertices in orbit $\mathrm{A}$ is the origin, as is the barycenter of the vertices in orbit $\mathrm{D}$. Moreover, the sum of the barycenter of vertices in orbit $\mathrm{B}$ and orbit $\mathrm{C}$ is as well the origin.

\section{ACKNOWLEDGEMENTS}

The authors would like to thank Christophe Hohlweg for bringing this open problem to their attention and for various discussions.

The authors had the idea for the solution at the Formal Power Series and Algebraic Combinatorics conference in Nagoya, Japan, in August 2012. They are grateful to the organizers for this wonderful meeting and for the financial support.

\section{REFERENCES}

[CFZ02] Frédéric Chapoton, Sergey Fomin, and Andrei Zelevinsky, Polytopal realizations of generalized associahedra, Dedicated to Robert V. Moody. Canad. Math. Bull. 45 (2002), no. 4, 537-566, DOI 10.4153/CMB-2002-054-1. MR.1941227 (2003j:52014)

[CLS11] Cesar Ceballos, Jean-Philippe Labbé, and Christian Stump, Subword complexes, cluster complexes, and generalized multi-associahedra, J. Algebraic Combin. 39 (2014), no. 1, 17-51, DOI 10.1007/s10801-013-0437-x. MR3144391

[FZ03a] Sergey Fomin and Andrei Zelevinsky, Cluster algebras. II. Finite type classification, Invent. Math. 154 (2003), no. 1, 63-121, DOI 10.1007/s00222-003-0302-y. MR2004457 (2004m:17011)

[FZ03b] Sergey Fomin and Andrei Zelevinsky, $Y$-systems and generalized associahedra, Ann. of Math. (2) 158 (2003), no. 3, 977-1018, DOI 10.4007/annals.2003.158.977. MR2031858 (2004m:17010)

[HL07] Christophe Hohlweg and Carsten E. M. C. Lange, Realizations of the associahedron and cyclohedron, Discrete Comput. Geom. 37 (2007), no. 4, 517-543, DOI 10.1007/s00454007-1319-6. MR2321739(2008g:52021) 
[HLR10] Christophe Hohlweg, Jonathan Lortie, and Annie Raymond, The centers of gravity of the associahedron and of the permutahedron are the same, Electron. J. Combin. 17 (2010), no. 1, Research Paper 72, 14. MR2651725 (2011f:05334)

[HLT11] Christophe Hohlweg, Carsten E. M. C. Lange, and Hugh Thomas, Permutahedra and generalized associahedra, Adv. Math. 226 (2011), no. 1, 608-640, DOI 10.1016/j.aim.2010.07.005. MR.2735770 (2012d:20085)

[Hoh12] Christophe Hohlweg, Permutahedra and associahedra: generalized associahedra from the geometry of finite reflection groups, Associahedra, Tamari lattices and related structures, Prog. Math. Phys., vol. 299, Birkhäuser/Springer, Basel, 2012, pp. 129-159, DOI 10.1007/978-3-0348-0405-9_8. MR3221538

[Hum78] James E. Humphreys, Introduction to Lie algebras and representation theory, Graduate Texts in Mathematics, vol. 9, Springer-Verlag, New York, 1978. Second printing, revised. MR499562 (81b:17007)

[Hum90] James E. Humphreys, Reflection groups and Coxeter groups, Cambridge Studies in Advanced Mathematics, vol. 29, Cambridge University Press, Cambridge, 1990. MR1066460 (92h:20002)

[KM04] Allen Knutson and Ezra Miller, Subword complexes in Coxeter groups, Adv. Math. 184 (2004), no. 1, 161-176, DOI 10.1016/S0001-8708(03)00142-7. MR2047852(2005c:20066)

[Lod04] Jean-Louis Loday, Realization of the Stasheff polytope, Arch. Math. (Basel) 83 (2004), no. 3, 267-278, DOI 10.1007/s00013-004-1026-y. MR.2108555(2005g:52028)

[PP12] Vincent Pilaud and Michel Pocchiola, Multitriangulations, pseudotriangulations and primitive sorting networks, Discrete Comput. Geom. 48 (2012), no. 1, 142-191, DOI 10.1007/s00454-012-9408-6. MR2917206

[PS11] Vincent Pilaud and Christian Stump, Brick polytopes of spherical subword complexes and generalized associahedra. To appear in Adv. Math. Preprint, arXiv:1111.3349, 2011.

[PS12] Vincent Pilaud and Francisco Santos, The brick polytope of a sorting network, European J. Combin. 33 (2012), no. 4, 632-662, DOI 10.1016/j.ejc.2011.12.003. MR2864447

[PS13] Vincent Pilaud and Christian Stump, EL-labelings and canonical spanning trees for subword complexes, Discrete geometry and optimization, Fields Inst. Commun., vol. 69, Springer, New York, 2013, pp. 213-248, DOI 10.1007/978-3-319-00200-2_13. MR.3156785

[Rea06] Nathan Reading, Cambrian lattices, Adv. Math. 205 (2006), no. 2, 313-353, DOI 10.1016/j.aim.2005.07.010. MR2258260 (2007g:05195)

[Rea07] Nathan Reading, Sortable elements and Cambrian lattices, Algebra Universalis 56 (2007), no. 3-4, 411-437, DOI 10.1007/s00012-007-2009-1. MR2318219 (2008d:20073)

[RS09] Nathan Reading and David E. Speyer, Cambrian fans, J. Eur. Math. Soc. (JEMS) 11 (2009), no. 2, 407-447, DOI 10.4171/JEMS/155. MR2486939(2011a:20102)

[Ste13] Salvatore Stella, Polyhedral models for generalized associahedra via Coxeter elements, J. Algebraic Combin. 38 (2013), no. 1, 121-158, DOI 10.1007/s10801-012-0396-7. MR.3070123

[Stu11] Christian Stump, A new perspective on $k$-triangulations, J. Combin. Theory Ser. A 118 (2011), no. 6, 1794-1800, DOI 10.1016/j.jcta.2011.03.001. MR2793610 (2012e:52042)

[Woo04] A. Woo, Catalan numbers and Schubert polynomials for $w=1(n+1) \ldots 2$. Preprint, arXiv:0407160, 2004.

CNRS and LiX, École Polytechnique, 91128 Palaiseau, France

E-mail address: vincent.pilaud@lix.polytechnique.fr

$U R L:$ http://www.lix.polytechnique.fr/ pilaud/

Institut für Algebra, Zahlentheorie, Diskrete Mathematik, Universität Hannover, Hannover, Germany

E-mail address: stump@math.uni-hannover.de

Current address: Diskrete Geometrie, Freie Universität Berlin, Arnimallee 2, 14195 Berlin, Germany

E-mail address: christian.stump@fu-berlin.de

URL: http://homepage.univie.ac.at/christian.stump/ 